for constants $a_{i}$ properly chosen. Conversely, for any choice of constants $a_{i}$ any solution of (6.3) is a solution of (6.2). If $\left(k, a_{0}\right)$ is in $R$ and (4.3) and (4.4) are satisfied, it can be shown by applying Theorem 2.1 that equation (6.3) has a unique solution on some interval $[k, k+h]$ and that

$$
y^{\alpha+i}(k)=a_{i}, \quad i=0, \cdots, p-2,
$$

where $y^{\alpha+i}$ is the derivative of order $\alpha+i$. This leads to the following theorem:

Theorem 6.1. If $\beta, \alpha, p$ are numbers as described above, if $\phi(x, y)$ satisfies (4.3) and (4.4), and if $a_{0}, a_{1}, \cdots, a_{p-2}$ is any set of numbers with $\left(k, a_{0}\right)$ in $R$, then the equation

$$
D_{x}^{\beta} y=\phi(x, y)
$$

has a unique solution satisfying the initial conditions (6.4).

HARVARD UNIVERSITY AND

Georgia School of Technology

\title{
NOTE ON INTEGRABILITY CONDITIONS OF IMPLICIT DIFFERENTIAL EQUATIONS*
}

\section{CLYDE M. CRAMLET}

The Riquier $\dagger$ theory for computing the integrability conditions of a system of partial differential equations of arbitrary order but in a special form gives a precise method for calculating these conditions without repetitions and for obtaining the initial determinations of the solutions. These general arguments imply a corresponding theorem for implicit systems of equations. It is the purpose of the present note to state that theorem and to point out that it is a consequence of the general theory. All references will be to the Janet exposition.

Let $F^{k},(k=1,2, \cdots, m)$, represent a system of differential equa-

* Presented to the Society, December 28, 1934.

†C. Riquier, Les Systèmes d'Équations aux Dérivées Partielles, Paris, 1910. M. Janet, Les systèmes d'équations aux dérivées partielles, Journal de Mathématiques, (8), vol. 3 (1920), pp. 65-151. J. M. Thomas, Riquier's existence theorems, Annals of Mathematics, vol. 30 (1929), pp. 285-310. J. F. Ritt, American Mathematical Society Colloquium Publications, vol. 14, chap. 9. 
tions of order $k$ in the $n$ independent variables $x$ and the $r$ dependent variables $z$. The chain of sets of equations

$$
F \equiv F^{0}, F^{1}, F^{2}, \cdots, F^{m}
$$

will be said to be complete if it has the following properties:

(a) The set $F^{0}$ contains and implies no equations independent of a $z$.

(b) The derivatives of the set $F^{k}$ are among the set $F^{k+1}$, $(k=0,1,2, \cdots, m)$.

(c) Any equations deducible from $F^{k}$ (integrability conditions) that are of order $s$ will be among the sets $F^{s},(s=0,1, \cdots, k ; k=1$, $2, \cdots, m)$.

(d) The equations of the set $F^{m+1}$ contain only derivatives of $F^{m}$ and no equations of type (c) are obtainable from $F^{m+1}$ except the equations already appearing in the sets $F^{s},(s=0,1, \cdots, m)$.

We shall prove the following theorem:

An integrable system is reducible to a complete system (1). A complete system is integrable.

The second part of the theorem will be proved first. Each step in the general theory may be carried out for the implicit system. For the purpose of the proof of the general existence theorem and for the determination of initial conditions it is necessary to classify the derivatives appearing in the equations. By the implicit function theorem the higher derivatives may be solved for, and by the method of Janet (chap. 2, \$12) the equations may be transformed to satisfy the Janet conditions (a) and (b) (chap. 2, §6). These derivatives appearing in the left members are for convenience associated with a set $M$ of monomials which are the basis for a module determining all principal derivatives.

If $m_{1}$ is any monomial of the module $M$, the product of $m_{1}$ by any non-multiplier will be a monomial $m_{2}$ belonging to the module. If $d_{1}$ is the derivative corresponding to $m_{1}$ which is the left member of an equation $e_{1}$, then $e_{2}$ will be obtainable from (1) by property (b). Consequently the module $M$ may be assumed to be complete. Hence all integrability conditions are in the set in consequence of property (c). The system (1) is, accordingly, integrable. The conditions under which the derivatives corresponding to the modulus $M$ may be solved for by the use of the implicit function theorem constitute additional restrictions under which the general existence theorem is applicable. Under these restrictions the initial conditions may be determined, from the knowledge of the monomials $M$. 
We have shown that (1) is integrable. It remains to demonstrate the possibility of the reduction of an integrable system $S$ to a complete system.

For this purpose we classify the derivatives in the original system $S$, as we have above, into the classes principal and parametric. The Riquier theory gives us the precise number of integrability conditions, which will be equal to the total number of independent equations obtainable from the derivatives of the set $S \equiv S^{0}, S^{1}, \ldots, S^{i}$. Let the equations obtained by differentiating these or any equivalent equations be designated by the sets $f^{0}, f^{1}, \cdots, f^{i+1}$. The sets of equations $f^{r}$ will be adjoined to the original equations of order $r$ to form a class $E^{r},(r=0,1, \cdots, i+1)$. In dealing with symmetrical sets of equations $f^{r}$ may be difficult to obtain or to deal with when obtained. It will be satisfactory to include in $E^{r}$ all results of differentiating $S^{r-1}$ once, together with all $r$ th order equations which are needed to insure the algebraic consistency in the principal derivatives of the derivatives of $S^{k},(k=r-1, \cdots, i)$.

This new set $E$ may be treated like the set $S$. This process must end in any case-in case of integrability or non-integrability. This is apparent from the observation that at each stage new monomials are added to a set which is a basis for a module and the fact that every module has a finite basis.

Accordingly we have the following theorem:

(a) If the set $F^{0}$ is vacuous, the equations (1) have a solution with the initial determination given by the Riquier theory.

(b) If the set $F^{0}$ contains any equations independent of all of the dependent variables, the equations are inconsistent.

(c) If the set $F^{0}$ contains only equations determining dependent variables $z$, these functions together with the solutions of the remaining independent equations and the initial determinations constitute the solutions.

UNIVERSITY OF WASHINGTON 